\title{
Micromachining with femtosecond laser written radial polarization converter
}

\author{
Martynas Beresna, Mindaugas Gecevičius and Peter G. Kazansky \\ Optoelectronics Research Centre, University of Southampton, SO17 1BJ Southampton, UK \\ Authore-mail address: mxb@orc.soton.ac.uk \\ Audrey Champion and Yves Bellouard \\ Eindhoven University of Technology, 5600 MB Eindhoven, the Netherlands
}

\begin{abstract}
Structures for microfluidics are fabricated with radially polarized femtosecond laser beam. Radial polarization is produced using birefringent optical element. Omnidirectional etching can be achieved using cylindrically symmetric polarization.
\end{abstract}

OCIS codes: (140.7090) Ultrafast lasers; (260.1440) Birefringence; (320.2250) Ultrafast phenomena

Femtosecond laser induced enhancement of etching rate in fused silica is of particular interest for micro-fluidic channel fabrication [1,2]. However, one of the main difficulties in this process is the strong etching rate dependence on the beam polarization related to ultrashort light pulse induced nanostructures [3]. Potential solution for this problem could be beams with radial or azimuthal polarization, which can induce radially symmetric nanometer-scale patterns on the surface of fused silica [6]. However, for wider and commercial applications an efficient and simple radial/azimuthal polarization generation or conversion technique is required. Recently, an effective polarization converter for the visible was fabricated by the means of ultra-fast laser direct writing [7]. In this paper we demonstrate that the same technique can be exploited for efficient polarization converters operating in the nearinfrared. The fabricated converters are employed in the ultra-fast laser writing setup to induce permanent modification in the bulk of fused silica. As a result we demonstrate that presented polarization converter can be exploited for the efficient micro-fluidic channel fabrication.

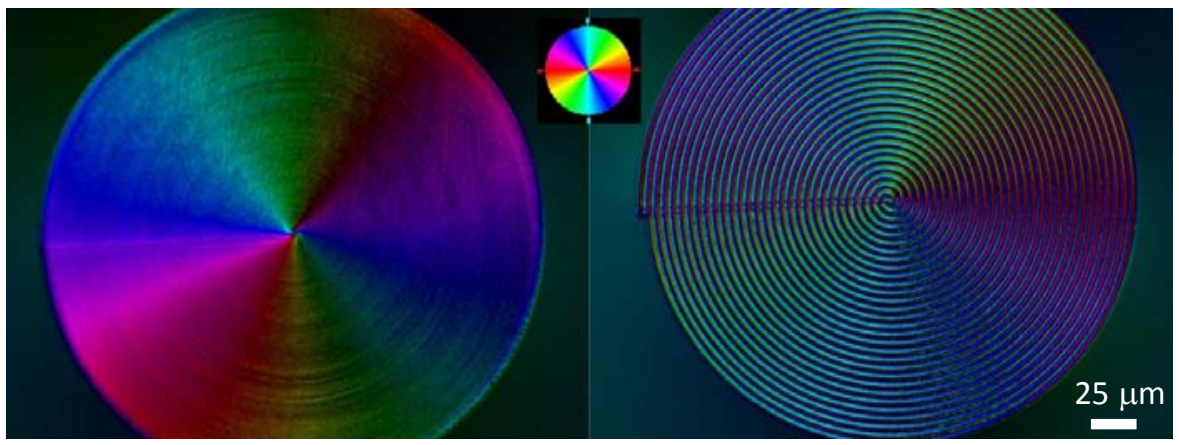

Fig. 1. Birefringent structures written with femtosecond radially polarized beam. Clear dependence of slow axis orientation on writing direction can be seen. Uniform slow axis orientation can be achieved overlapping adjacent lines (Left), if the interline distance is increased each laser written has a complex slow axis variation across the line.

For imprinting the polarization the beam from femtosecond laser system PHAROS (LightConversion Ltd.) was focused with an aspheric lens $(\mathrm{NA}=0.16)$. The sufficient level of retardance was achieved by consecutively writing two birefringent layers of about $257 \mathrm{~nm}$ retardance separated by $30 \mu \mathrm{m}$. The computer controlled translation stages (Aerotech Inc.) were programmed with SCA software (Altechna Ltd., Lithuania) to move the sample in a spiral trajectory with steps of $2 \mu \mathrm{m}$, so that in a complete scan the writing laser beam would uniformly cover a circular area of diameter $6 \mathrm{~mm}$. Total fabrication time for the structure was 8 hours.

The fabricated polarization converter was integrated into ultrafast laser writing set-up to generate cylindrically symmetric polarization state. Two structures of different inter-line separation $(1 \mu \mathrm{m}$ and $5 \mu \mathrm{m})$ were written with a radially polarized femtosecond laser beam (Fig. 1). The circular areas where covered by moving the sample in spiral trajectory towards the centre of the structure. No additional polarization control was included. The pulse energy was set to the regime where nanogratings are known to be formed. After the irradiation the samples were inspected with a quantitative birefringence measurement system Abrio (CRi Inc.). The slow axis of the induced birefringence clearly follows direction of the scanning, indicating that nanogratings are spontaneously orienting with scanning direction. If the inter-line distance is smaller than the spot size of the scanning laser a uniform radial distribution of 
the slow axis can be achieved (Fig. 1 left). It is known that femtosecond laser induced nanograting records the last polarization state of the laser beam, thus effectively it is acting as a rewritable data storage [8]. Due to this re-writing phenomenon only central part will retain its nanograting orientation related to translation direction of the writing beam. In practice this effect can significantly simplify fabrication of polarization sensitive devices eliminating need of additional polarization control [7].
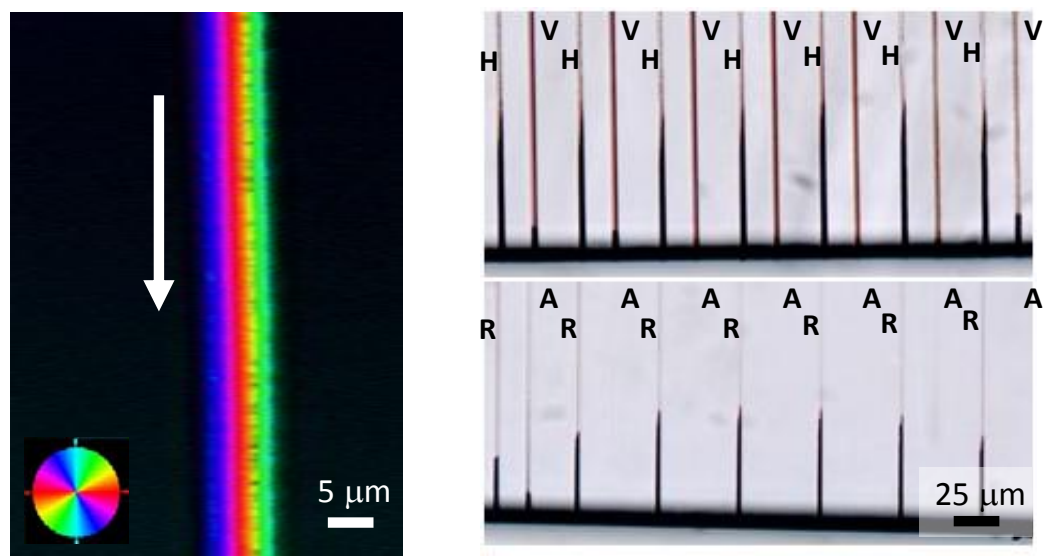

Fig. 2. (Left) The Abrio image of a line written with radial polarization. White arrow indicates writing direction. (Right) Structures inscribed with linear: horizontal $(\mathrm{H})$ and vertical $(\mathrm{V})$ and cylindrical: radial (R) and azimuthal (A) polarizations. One can see that similar etching ( $1.5 \mathrm{~h}$ etching in diluted HF) results can be achieved with horizontal and radial polarizations.

In the next experiment, we attempted to compare structures written with linear and radial/azimuthal polarizations. A set of lines were written with constant average power at several translation speeds. After inscription the structures were investigated under polarization microscope. The color variation across the laser written line indicates that nanograting direction is changing perpendicular to the writing direction (Fig. 2, left). Additionally, different color distribution can be seen for radial and azimuthal polarizations thus excluding possibility of stress induced birefringence, which also appear in similar direction dependent character. The inscribed lines were subsequently etched with diluted HF. One can see that azimuthal polarization performs almost as well as horizontal linear polarization.

Currently, we are investigating application of azimuthal/radial polarizations for fabrication of micro-fluidic channels in fused silica glass.

\section{Acknowledgment}

The work was supported the project FEMTOPRINT, financed by the European Commission Factories of the Future program (FP7/ NMP/Project No 260103), http://www.femtoprint.eu/

\section{References}

1. Y. Bellouard, A. Said, M. Dugan, and P. Bado, "Fabrication of high-aspect ratio, micro-fluidic channels and tunnels using femtosecond laser pulses and chemical etching," Optics express 12, 2120-9 (2004).

2. A. Marcinkevičius, S. Juodkazis, M. Watanabe, M. Miwa, S. Matsuo, H. Misawa, and J. Nishii, "Femtosecond laser-assisted threedimensional microfabrication in silica," Optics Letters 26, 277 (2001).

3. C. Hnatovsky, R. S. Taylor, E. Simova, V. R. Bhardwaj, D. M. Rayner, and P. B. Corkum, "Polarization-selective etching in femtosecond laser-assisted microfluidic channel fabrication in fused silica," Optics Letters 30, 1867 (2005).

4. R. Dorn, S. Quabis, and G. Leuchs, "Sharper focus for a radially polarized light beam," Physical Review Letters 91, (2003).

5. V. G. Niziev and A. V. Nesterov, "Influence of beam polarization on laser cutting efficiency," Journal of Physics D: Applied Physics 32, 1455-1461 (1999).

6. C. Hnatovsky, V. Shvedov, W. Krolikowski, and A. Rode, "Revealing local field structure of focused ultrashort pulses," Physical Review Letters 106, 1-4 (2011).

7. M. Beresna, M. Gecevičius, P. G. Kazansky, and T. Gertus, "Radially polarized optical vortex converter created by femtosecond laser nanostructuring of glass," Applied Physics Letters 98, 201101 (2011).

8. Y. Shimotsuma, M. Sakakura, P. G. Kazansky, M. Beresna, J. Qiu, J. Qiu, K. Miura, and K. Hirao, "Ultrafast manipulation of selfassembled form birefringence in glass," Advanced materials 22, 4039-43 (2010). 